\title{
An investigation on human dynamics in enclosed spaces
}

\author{
Marcello Trovati \\ Department of Computer Science, Edge Hill University, UK \\ Eleana Asimakopoulou \\ Hellenic National Defence College \\ Greece \\ Nik Bessis \\ Department of Computer Science, Edge Hill University, UK
}

\begin{abstract}
In this article, we introduce a method for analysing specific dynamical properties associated with the movement of people on a two-dimensional (compact) space. We focus on a variety of features defined by the topology and dynamics of the system to investigate human dynamics in enclosed spaces. This can potentially have significant applications within systems defined by human behaviour, particularly relevant to disaster management, internet of things (IoT), and big data analytics.

Keywords: Mathematical modelling, human behaviour, disaster management, internet of things
\end{abstract}

\section{Introduction}

Human behaviour typically exhibits complex features, which have been investigated via various models and simulation techniques, where the aim is to determine the most important factors in the time and the way of reaching a specific target, such as exiting a room [1].

In this article, we propose an investigation of the dynamical properties of systems defined by the movement of people over a two-dimensional (compact) space. Our approach is based on the properties of the network generated by 
specific parameters linking the different individuals, such as the mutual distance and leadership properties exhibited by some of them, which might influence the followers-leaders structure.

In particular, the choice of following a specific individual depends on global and local topological, as well as dynamical properties associated with the corresponding network, such as the connectedness of the different nodes and how this changes over time. Such properties have a direct impact on the overall behaviour of the system.

\subsection{Applications to IoT, big data and disaster management}

Internet of things (IoT) has been attracting increasing attention from the research community [2], [3]. In particular, the inter-connections among devices, buildings, sensors, etc. enable the collection and exchange of an enormous quantity of data, creating significant opportunities, as well as new challenges. Therefore, when IoT is integrated with big data technology, this can identify new trends and actionable information, which can be used in a variety of contexts and applications [4], [5]. In particular, disaster management is an important application, where IoT sensing and communication technologies can be utilised to monitor and gather data, even in the absence of any existing infrastructure [6].

The method proposed in this article can be used to investigate and assess human movement in enclosed spaces, by analysing the dynamical and selforganising properties of the corresponding system. Throughout this work, we assume that a suitable IoT framework, gathering relevant information regarding the positions of the corresponding individuals, is in place and fully functioning. The novelty of this article stems from its integration of various theoretical techniques with specific applications. In particular, the former allow a more comprehensive description of such systems, which has not previously been fully exploited. Furthermore, this work is part of a wider line of inquiry aiming to create a model based on a topological and algebraic framework. This will enable the identification and assessment of the macro and micro properties of the sys- 
tem, by using an efficient and agile computational approach. Furthermore, the mathematical formalism utilised in this research can be extended to integrated theoretical approaches currently developed in other contexts [7]. This will have important applications within disaster management, big data analytics, as well as various IoT scenarios.

The article is structured as follows: section 2 discusses the current research in this field, and section 3 introduces the main concepts and definitions used in this context. Sections 4 and 5 discuss the components of the model and the simulation results, respectively. Finally, section 6 concludes the article and discusses future research directions.

\section{Related work}

In [8], a model predicting the emergence of self-organisation phenomena is introduced. The authors investigate the combination of pedestrian heuristics with body collisions potentially creating crowd turbulence, based on an integrated assessment of simultaneous interactions between multiple individuals. In [9] a method for investigating realistic models of crowd dynamics is proposed, which considers an agent-based approach combined with non-homogeneous and asynchronous cellular automata. The authors argue that this approach enables the modelling of pedestrians' dynamics in various and complex environments. In [10], via the utilisation of exosomatic visual architecture, the authors show the feasibility of behavioural models where movement rules are based on Gibson's principle of affordance [11]. More specifically, they apply agents defined by such rules to a built-environment scenario, via the variation of specific parameters, such as destination selection, field of view, and steps taken between decision points.

The properties related to the behaviour of ants and pedestrians are investigated in [12], where the pheromone left by preceding ants creates a trail, which is followed by other ants. In a similar manner, pedestrians attempt to follow other 
individuals in a crowded environment to optimise efficient and safe walking. The authors propose a stochastic model, which integrates this type of behaviour based on local update rules. In particular, the relation between the ant trail model and the floor field model for studying evacuation dynamics of pedestrians is investigated, based on a two-dimensional generalisation of the ant trail model, where the pheromone is replaced by footprints. Their evaluation demonstrates that small perturbations will sometimes avoid congestion and hence allow safe evacuation.

A method for identifying human behaviour in a social network is introduced in [13], which is based on Markov chains trained on a specific number of models of normal human behaviour from social network data. This is subsequently, integrated with an activity detection framework, to identify unexplained activities on the basis of the normal behaviour models.

In [14], the authors discuss a model for the social interactions of pedestrians to enhance the prediction of their destination. An investigation of the phenomenon of the leader-follower behaviour is also provided, which is defined as the adjustment of an individual's motion to follow a leader.

In our proposed method, specific dynamical properties are emphasised, which can be easily expanded to address the interactions in human behaviour. These, in turn can be utilised to build a more robust theory based on topological and algebraic properties, as discussed in section 1.

\section{Main definitions and assumptions}

In this work, we consider an enclosed two dimensional space, such as a room, where a group of individuals move based on specific rules, aiming to eventually leave through one or more exits. In particular, their mutual relationships create a complex system, which evolves over time and is highly dynamic by nature. Figure 1 depicts an example of such scenario.

As discussed in section 2, there is extensive research on this topic, and it has 


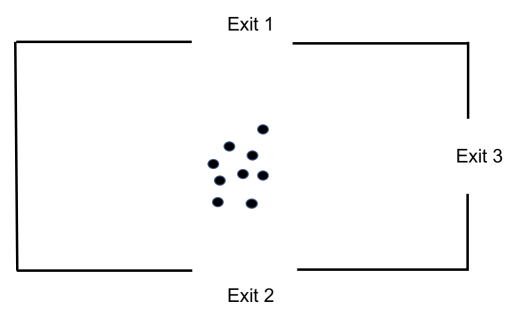

Figure 1: An example of the scenario described in section 3.

been demonstrated that IoT provides a framework to gather relevant data in this context [15]. In this article, we assume that the positions of the different individuals and any other information related to their environment, are gathered via a set of sensors and devices suitably positioned.

Network theory has been extensively investigated, and it has been shown to have numerous applications in several scenarios. In particular, networks can successfully model complex systems where the interactions among their components play an important role [16], [17]. More formally, a set of $n$ people (or nodes) can freely move according to some pre-defined rules over a twodimensional compact space $\mathcal{R}$, where each individual either follows (and/or is followed by other ones), or moves towards a target position, such as an exit. This type of interaction naturally defines a directed network $G=G(V, E)$, where $V=\left\{x_{i}\right\}_{i=1}^{n}$ is the node-set, and $E=\left\{\left(x_{i}, x_{j}\right)\right\}_{i \neq j=1}^{n}$ is the set containing all the pairs of nodes, where $x_{i}$ moves towards $x_{j}$.

For $x \in V$, let $n(x)=\{z \in V:(x, z) \in E\} \subset V$, that is the set of nodes adjacent to $x$, or its neighbours. Given that people are more likely to follow nearby individuals, the existence of an edge between two nodes also depends on their mutual (Euclidean) distance $d(\cdot, \cdot)$, which must be within a minimum value. However, this may not be the case in general, as there are other factors that influence such choice, as discussed later on in the article.

More specifically, we assume that each node can perform two possible actions:

1. $x$ follows another node $y \in n(x)$, i.e. one of its neighbours, or 
2. $x$ moves independently according to a pre-defined target position.

Most of all the nodes are assumed to perform the first action as otherwise, the system might not exhibit relevant self-organising properties leading to a trivial configuration. Furthermore, depending on the dynamics of the system some nodes may complete their itinerary after a finite number of iterations, whereas others might be "trapped" indefinitely.

With a slight abuse of notation, $x$ will also refer to the two-dimensional position of the corresponding node. At each time iteration, a node will move based on a set of translations $T_{i}: \mathcal{R} \rightarrow \mathcal{R}$, for $i=1,2,3$, where

$$
\begin{array}{r}
T_{1}(x, y)=x+\alpha \hat{u}_{(x, y)} \\
T_{2}(x, e x i t)=x+\alpha \hat{u}_{(x, e x i t)} \\
T_{3}(x, y \rightarrow e x i t)=x+\alpha \hat{u}_{(x, y \rightarrow e x i t)}
\end{array}
$$

where $\alpha$ is a scalar and $\hat{u}_{(a, b)}$ is the unit vector in the direction $\overrightarrow{a b}$. In particular,

- $T_{1}$ moves $x$ towards another node $y$. In this case $x$ is the follower and $y$ is its leader, and there is a directed edge $(x, y)$.

- Under $T_{2}, x$ does not follow any other node, as it moves towards one of the exits, and

- Finally, under $T_{3}, x$ "observes" $y$ moving towards an exit, and it will follow it, independently of any other constraint, such as their distance. In this case a directed edge must also be present. Loosely speaking, $x$ may choose to follow a more distant node if it is closer to one of the exits.

The iteration of the translations $T_{1}, T_{2}$ and $T_{3}$ naturally create a dynamical system over the network $G$, where mutual connection typically change over time, and exiting nodes are removed for the node set. We denote $G_{t}=G_{t}\left(V_{t}, E_{t}\right)$ to be the network $G$ at time $t$, where $V_{t+1} \subset V_{t}$. In particular, the choice of any of the above translations will depend not only on the distance between the nodes, but also on the weight of their mutual relationships as discussed in section 4 . 
In a real-world scenario, there might be areas that need to be avoided. Therefore, we define a no area $N_{a} \subset \mathcal{R}$ as a (compact) subspace, where nodes are not allowed. More specifically, if $x \notin N_{a}$ but its trajectory under $T_{i}$ intersects $N_{a}$, that is the line segment $\left[x, T_{i}(x)\right] \cap N_{a} \neq \emptyset$, then $x$ will follow a different translation so that it will not enter $N_{a}$.

As discussed above, nodes might follow another node if their corresponding translation will move them sufficiently close, or in other words their distance is within a specific threshold $D$. The following lemma provides a simple result based on this observation.

Lemma 1. Let $v_{t}$ and $y_{t}$ be two nodes at time $t$, which follow the nodes $z$ and $k$, respectively. In other words, $v_{t}=v_{t-1}+\alpha \hat{u}_{\left(v_{t-1}, z\right)}$ and $y_{t}=y_{t-1}+\alpha \hat{u}_{\left(y_{t-1}, k\right)}$, where $\hat{u}_{\left(v_{t-1}, z\right)}=\cos \theta_{1} \hat{i}+\sin \theta_{1} \hat{j}$ and $\hat{u}_{\left(y_{t-1}, k\right)}=\cos \theta_{2} \hat{i}+\sin \theta_{2} \hat{j}$. Assume $D$ is the minimum distance between $v_{t}$ and $y_{t}$ to have an edge between them.

Then $\left(v_{t}, y_{t}\right) \in E_{t}$ if

$$
\theta_{1}=\arctan \left(\frac{\left(v_{t-1}-z\right)_{y}}{\left(v_{t-1}-z\right)_{x}}\right) \text { and } \theta_{2}=\arctan \left(\frac{\left(y_{t-1}-k\right)_{y}}{\left(y_{t-1}-k\right)_{x}}\right),
$$

where $(\cdot)_{x}$ and $(\cdot)_{y}$ refer to the $x$ and $y$ coordinates, respectively.

Proof. Let $v_{t}=v_{t-1}+\alpha \hat{u}_{\left(v_{t-1}, z\right)}$ and $y_{t}=y_{t-1}+\alpha \hat{u}_{\left(y_{t-1}, k\right)}$ be two nodes moving towards $z$ and $k$, respectively. Assume that their distance $d\left(v_{t-1}, y_{t-1}\right)=$ $\left|v_{t-1}-y_{t-1}\right| \gamma$. Then, if $\hat{u}_{\left(v_{t-1}, z\right)}=\cos \theta_{1} \hat{i}+\sin \theta_{1} \hat{j}$ and $\hat{u}_{\left(y_{t-1}, k\right)}=\cos \theta_{2} \hat{i}+$ $\sin \theta_{2} \hat{j}$, then equation 6 can be written as

$\left|v_{t}-y_{t}\right|=v_{t-1}+\alpha \hat{u}_{\left(v_{t-1}, z\right)}-y_{t-1}+\alpha \hat{u}_{\left(y_{t-1}, k\right)} \leq\left|v_{t-1}-y_{t-1}\right|+\alpha\left|\hat{u}_{\left(v_{t-1}, z\right)}-\hat{u}_{\left(y_{t-1}, k\right)}\right|$

This is equal to

$$
\gamma+\alpha\left|\hat{u}_{\left(v_{t-1}, z\right)}-\hat{u}_{\left(y_{t-1}, k\right)}\right|
$$


If $\hat{u}_{\left(v_{t-1}, z\right)}=\cos \theta_{1} \hat{i}+\sin \theta_{1} \hat{j}$ and $\hat{u}_{\left(y_{t-1}, k\right)}=\cos \theta_{2} \hat{i}+\sin \theta_{2} \hat{j}$, then equation 6 can be written as

$$
\begin{array}{r}
\gamma+\alpha\left|\left(\cos \theta_{1}-\cos \theta_{2}\right) \hat{i}+\left(\sin \theta_{1}-\sin \theta_{2}\right) \hat{j}\right|= \\
\gamma+\alpha \sqrt{\left(\cos \theta_{1}-\cos \theta_{2}\right)^{2}+\left(\sin \theta_{1}-\sin \theta_{2}\right)^{2}}= \\
\gamma+\sqrt{2} \alpha \sqrt{1-\cos \theta_{1} \cos \theta_{2}-\sin \theta_{1} \sin \theta_{2}} .
\end{array}
$$

Let $D$ be the threshold so that if $d\left(v_{t-1}, y_{t-1}\right) \leq D$ then there is an edge between $v_{t}$ and $y_{t}$. Then we have that

$$
\begin{gathered}
\sqrt{1-\cos \theta_{1} \cos \theta_{2}-\sin \theta_{1} \sin \theta_{2}} \leq \frac{D-\gamma}{\sqrt{2} \alpha} \Rightarrow \\
\cos \theta_{1} \cos \theta_{2}-\sin \theta_{1} \sin \theta_{2} \geq 1-\frac{(D-\gamma)^{2}}{2 \alpha^{2}} .
\end{gathered}
$$

This yelds

$$
\theta_{1}=\arctan \left(\frac{\left(v_{t-1}-z\right)_{y}}{\left(v_{t-1}-z\right)_{x}}\right) \text { and } \theta_{2}=\arctan \left(\frac{\left(y_{t-1}-k\right)_{y}}{\left(y_{t-1}-k\right)_{x}}\right),
$$

where $(\cdot)_{x}$ and $(\cdot)_{y}$ refer to the $x$ and $y$ coordinates, respectively.

\section{Description of the method}

From a general network $G_{t}=G_{t}\left(V_{t}, E_{t}\right)$, we might have the following two scenarios: every node might be equally likely to follow any of its neighbours, that is

$$
p_{t}(x \rightarrow y)=\frac{1}{\operatorname{deg}(x)},
$$

for $x \in V$ and $y \in n_{t}(x)$, or there might be a specific hierarchy characterising the overall dynamics. There is evidence that any type of influence between nodes in a real-world network depends on its topological properties [17]. In this article, we assume that

$$
p_{t}(x \rightarrow y)=\frac{\operatorname{deg}(x)}{\sum_{y_{k} \in n_{t}(x)} \operatorname{deg}\left(y_{k}\right)} .
$$

In other words, the probability of following $y$ depends on the number of its neighbours and how well they are connected. As a consequence, equation 11 
also gives the probability that a translation has direction $\hat{u}_{(x, y)}$.

The trivial case, resulting in a complete network, is when all nodes are equally probable to follow any other node in the systems, which is equivalent to

$$
p_{t}(x \rightarrow y)=\frac{1}{\left|V_{t}\right|-1} .
$$

Note that, from equations 11 and 10, it follows that a random choice of the neighbours of a node is equivalent to them having the same degree. In fact

$$
\begin{aligned}
\frac{1}{\operatorname{deg}(x)} & =\frac{\operatorname{deg}(x)}{\sum_{y_{k} \in n_{t}(x)} \operatorname{deg}\left(y_{k}\right)} \\
& \Rightarrow \operatorname{deg}(x)=\operatorname{deg}\left(y_{k}\right)=n \in \mathbb{N} .
\end{aligned}
$$

Let $x \in V_{t}$ and $y \in n_{t}(x)$.

Definition 1. Define the weight of the edge $(x, y)$ at the time $t$ as

$$
w_{t}(x, y)=f_{t}(x, y) \circ p_{t}(x \rightarrow y)
$$

for a function $f_{t}(x, y)$.

The behaviour of the weight of the edges described by equation 13 is characterised by function $f_{t}(x, y)$. As discussed in section 3 , the distance between nodes is one of the factors that influences the overall topological structure of the network $G$. However, this is not always the case, as the node $x$ might follow another route based on the translations $T_{1}, T_{2}$ and $T_{3}$. Furthermore, a bias in the decision-making process (when choosing a route to follow) might be present. Therefore, we define $f_{t}(x, y)$ as

$$
f_{t}(x, y)=\delta_{x, y} e^{-\tilde{d}_{t}(x, y)},
$$

where $\delta_{x, y} \in[0,1]$ is a perturbation value, which can be used to model the decision of the node to follow another route based on a decision bias. Finally, let $\tilde{d}_{t}(x, y) \in[0,1]$ be the normalised distance at time $t$ calculated as

$$
\tilde{d}_{t}(x, y)=\frac{d_{t}(x, y)}{\max _{a, b \in V_{t}}\left\{d_{t}(a, b)\right\}} .
$$

Therefore, by combining equations 13 and 14, we have that

$$
w_{t}(x, y)=\frac{\delta_{x, y} e^{-\tilde{d}_{t}(x, y)} \operatorname{deg}(x)}{\sum_{y_{k} \in n_{t}(x)} \operatorname{deg}\left(y_{k}\right)}
$$




\subsection{Definition of leadership}

In this article, we assume that the level of leadership exhibited by a node $x$ depends on the number of other nodes following it, based on the dynamics of the whole system. More specifically, its leadership is impacted by its level of connectedness with its followers, in terms of the overall weight. However, the different weights vary with respect to time as described by (16). As a consequence, the time variation of the leadership of the node $x$ must be considered to provide an accurate insight into its behaviour, as described in the following definition.

Definition 2. The level of leadership $l_{t}(x)$ of a node $x$ is defined as the average weight of its followers based on equation 11 at a specific time $t$, that is

$$
l_{t}(x)=\frac{1}{\left|n_{t}(x)\right|} \sum_{y \in n_{t}(x)} w_{t}(x, y)
$$

In order to capture the different leadership time snapshots, let $L_{k}(x) \subset V^{k}$ such that

$$
L_{k}(x)=\left(l_{1}(x), \ldots, l_{k}(x)\right), \quad \text { where } \quad l_{i}=\frac{1}{\left|n_{i}(x)\right|} \sum_{y \in n_{i}(x)} w_{i}(x, y) .
$$

In particular, this provides information on the overall level of leadership of the node $x$ over the time $t=1, \ldots, k$.

Algorithm 1, describes the action of the different translations $T_{1}, T_{2}$ and $T_{3}$ for a node $x$, under the conditions discussed above. 


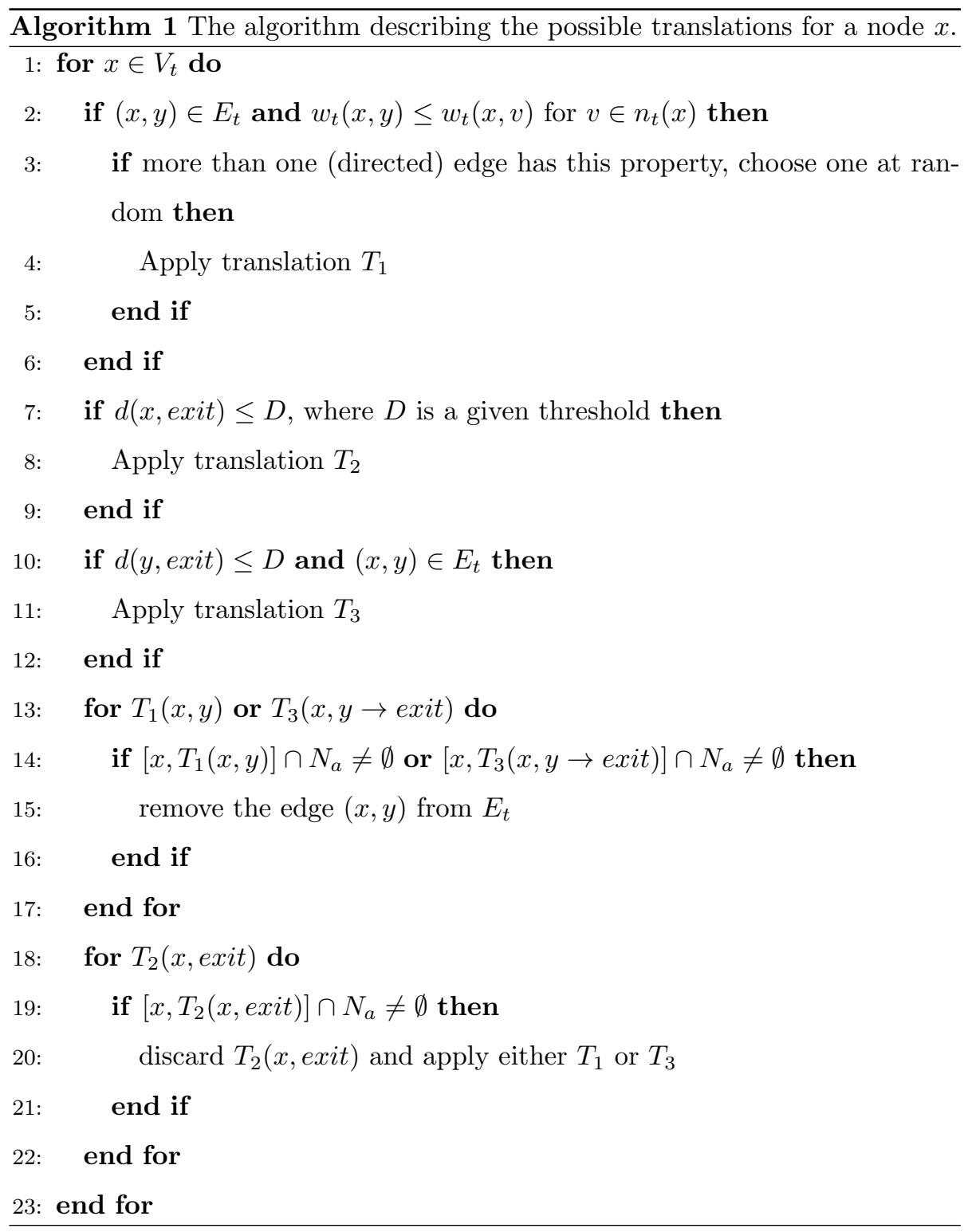

\subsection{Disorder of the system}

In the general scenario described above, the behaviour of the nodes impacts the overall stability of the system, in terms of reaching the optimal solution, that is all nodes have exited the space. In particular, the level of disorder of the system depends on the level of leadership exhibited by some specific nodes, 
as well as on how quickly the exit $\mathcal{R}$. Loosely speaking, the fastest and more orderly the space $\mathcal{R}$ is vacated, the more optimal the solution is. For example, if the overall network behaviour is purely random, a node is equally likely to choose any of its neighbours, and no clear leadership among them might be present.

Definition 3. We define the disorder $D_{t}(x, v)$ between two connected nodes $x$ and $v$, and the disorder $D_{t}(x)$ of node $x$ at time $t$ as

$$
D_{t}(x, v)=-\log \left(\frac{w_{x, v}}{\sum_{y \in n(x)} w_{x, y}}\right)
$$

and

$$
D_{t}(x)=\sum_{v \in n(x)}-\log \left(\frac{w_{x, v}}{\sum_{y \in n(x)} w_{x, y}}\right),
$$

respectively.

Let $\mathbf{D}_{\mathbf{k}}(x, v)=\left(D_{t}(x, v)\right)_{t=1}^{k}$ and $\mathbf{D}_{\mathbf{k}}(x)=\left(D_{t}(x)\right)_{t=1}^{k}$ be the set of disorder values between $x$ and $v$ and the overall node $x$ over $t=1, \ldots, k$, respectively.

Furthermore, from equation 17

$$
D_{t}(x, v)=-\log \left(\frac{w_{t}(x, v) \operatorname{deg}(x)}{l_{t}(x, v)}\right),
$$

which shows that a node with a strong level of leadership is associated with low disorder, if it has followers with weight close to 1 . In order to assess the overall behaviour of the network, we need to expand Definition 3 to consider all the nodes $x \in V_{t}$

Definition 4. We define the global disorder $D_{t}(G)$ for the whole network $G$ as

$$
D_{t}\left(G_{t}\right)=\sum_{x \in V_{t}} D_{t}(x)=\sum_{x \in V_{t}} \sum_{v \in n_{t}(x)}-\log \left(\frac{w_{t}(x, v)}{\sum_{y \in n_{t}(x)} w_{t}(x, y)}\right),
$$

and let $\mathbf{D}_{\mathbf{k}}\left(G_{t}\right)=\left(D_{t}\left(G_{t}\right)\right)_{t=1}^{k}$.

Assuming we are aiming for a higher level of leadership, and as a consequence a lower level of disorder, the above can be used to identify the most appropriate 
leaders over the network $G$. In this article, we will assess the overall behaviour of the system by considering each of the $\mathbf{D}_{\mathbf{t}}\left(G_{t}\right)$, for $t=1, \ldots, k$, as discussed in the next section. Also, note that $\mathbf{D}_{\mathbf{t}}\left(G_{t}\right) \geq \mathbf{D}_{\mathbf{t}+\mathbf{1}}\left(G_{t+1}\right)$, as each time a node exits $\mathcal{R}$ the disorder of the network decreases.

\section{Simulation results}

In this section, the simulation results are discussed, which consist of a variety of components. More specifically, Matlab and Python were used to evaluate and visualise the results.

In [18] (table 1, page 7), the authors discuss different experimental settings used in crowd behaviour modelling techniques. Although our evaluation does not focus on a bespoke dataset, we believe it demonstrates the potential of our approach. More specifically, this can be applied to both structured and unstructured data. In future research, we are aiming to provide a comprehensive experimental comparison with existing methods, based on specific data collected from various sources.

\subsection{Preliminary evaluation}

First of all, we defined a network $G$, with 100 nodes, randomly connected via edges, whose positions were uniformly distributed and equally distant along a circle. In particular, no exits and no areas were considered at this stage. Subsequently, we assumed $\alpha=0.1, \delta(x, y)=1$ over an iteration time $t=$ $1, \ldots, 100$. Clearly, the implementation of the model did not lead to any major change in the topology of the network as the nodes mainly moved along the circumference, as depicted in figure 2. Subsequently, we defined two random nodes $x_{1}=(0,-1)$ and $x_{2}=(0,0)$ as leaders, whose target positions were $(1,1)$ and $(0.75,0.75)$, respectively. In this case, we set $\delta\left(y, x_{1}\right)=\delta\left(z, x_{2}\right)=1$ for all $y$ and $z$ in $n\left(x_{1}\right)$ and $n\left(x_{2}\right)$, respectively. For all the other nodes $a, b \in V \backslash\left\{x_{1}, x_{2}\right\}$ such that $(a, b) \in E$, then $\delta(a, b)=0.5$. Figure 2 depicts their final positions. 

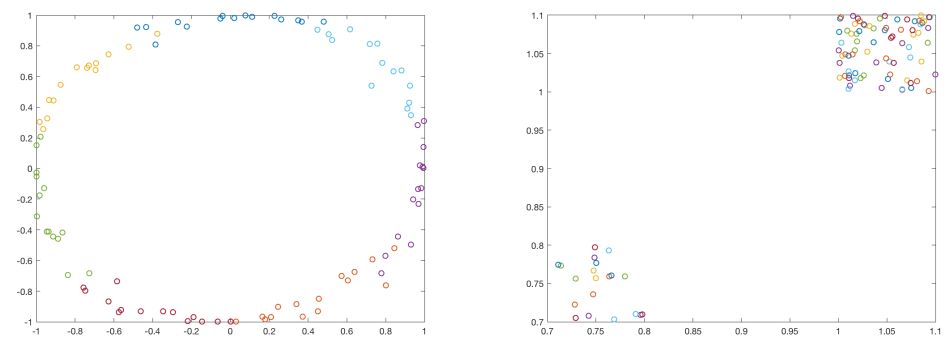

Figure 2: The left-hand side figure depicts the initial position of the points as described in section 5. The right-hand side shows the final position of the points, where $\alpha(x, y)=0.1$ and $\delta(x, y)=1$ for all $(x, y) \in E$.

Subsequently, we defined a small network with 10 nodes as depicted in figure 3 , clearly showing the existence of two potential leaders, namely node 2 and node 6 . The model described above was implemented with "no bias" over 50 iterations, that is with the same parameter $\delta_{x, y}$ for all nodes $x$, and $y$, such that $(x, y) \in E$. As depicted in figure 3, the network topology shows that node 6 is now the leader. Finally, we ran the same simulation, but in this case the bias was towards node 5 , that is $\delta(x$, node 5$)=1$, for all $x \in n$ (node 5$)$, and $\delta(a, b)=0.3$ for all the other linked nodes $a, b \in V \backslash\{$ node 5$\}$.

Although node 5 did not exhibit any strong leadership at first in terms of number of its connections, at the end of the iterative steps, it is strongly connected, as shown in figure 3 .

Furthermore, figure 4 shows the values of $l_{i}$ (node 5 ) over the iterative process.

We then considered a similar network as in the first part with 100 nodes but uniformly distributed and randomly connected. In a similar fashion, two random nodes $z_{1}$ and $z_{2}$ were randomly selected with target positions $(0.75,0.15)$ and $(0.25,0.3)$, respectively. In this case $\delta\left(x, z_{1}\right)=1, \delta\left(x, z_{2}\right)=0.7$ and $\delta(a, b)=0.3$ for all the other nodes $(a, b) \in E$. As expected, $z_{1}$ had more followers than $z_{2}$, 

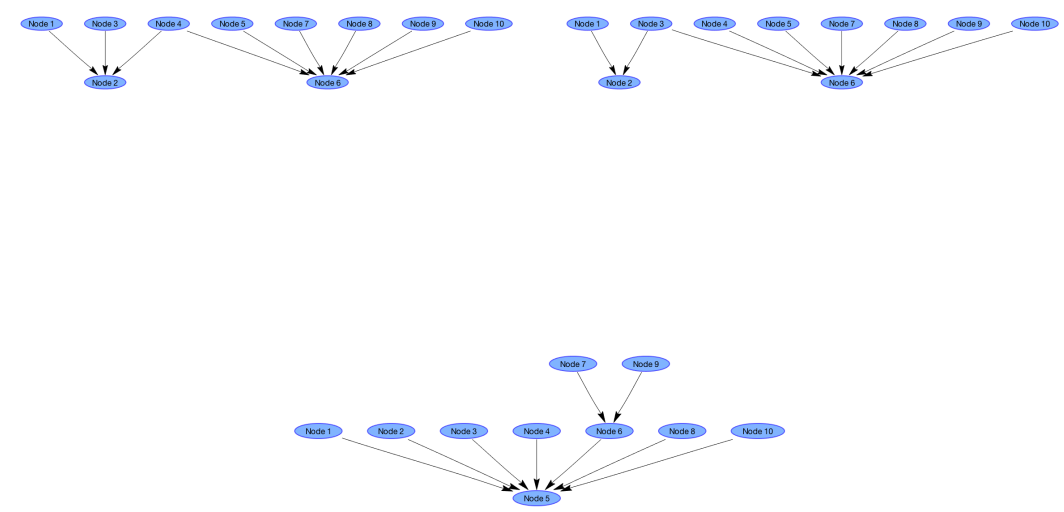

Figure 3: The initial network as described in section 5, the final network structure with no bias, and bias, respectively.

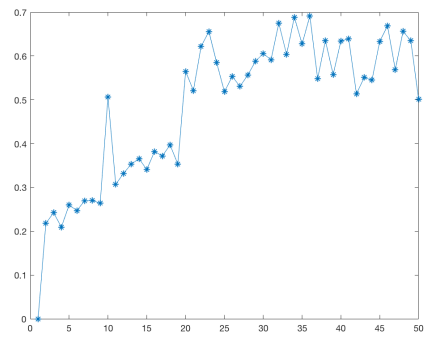

Figure 4: The values of $l_{k}$ (node 5$)$, for $k=1 \ldots, 50$.

as depicted in figure 5 .

\subsection{Evaluation with exits and no areas}

In the second part of the validation, we considered a space $\mathcal{R}=[0,1] \times[0,1]$, with three exits as depicted in figure 6

We generated three networks with 100 nodes randomly distributed and applied iteratively $T_{1}, T_{2}$, and $T_{3}$ with $\alpha=0.1, \delta(x, y)=1$, for all $(x, y) \in E_{t}$, as 

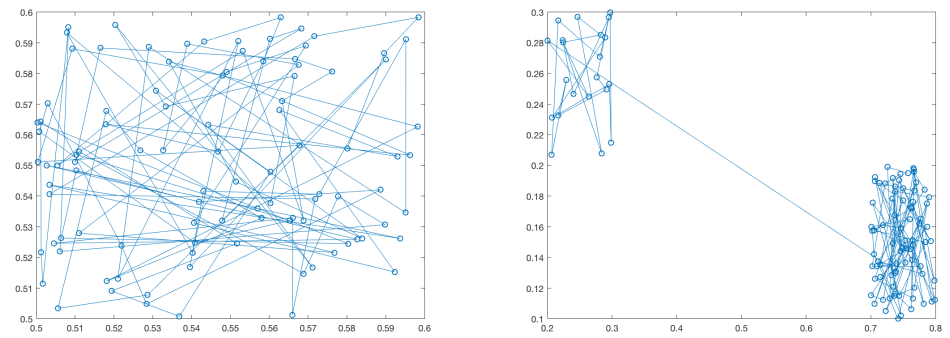

Figure 5: The network discussed in the final component of the simulation.

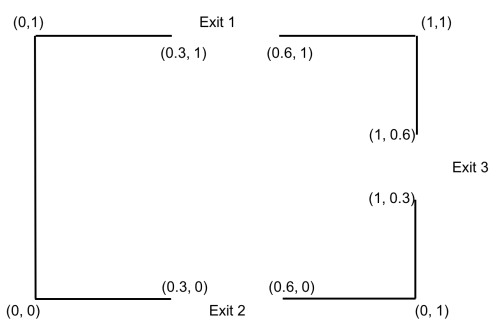

Figure 6: The room described in section 5.2.

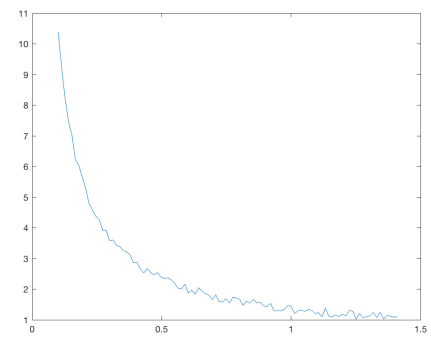

Figure 7: The average vacating iteration time versus different values of the minimum distance $D$.

discussed in algorithm 1.

Figure 7 shows the average time of exit for the networks, i.e. when all the nodes have left $\mathcal{R}$, with respect to their minimum distance $D$, as described in algorithm 1 . As expected, for $D \approx 0$, the nodes remain in $\mathcal{R}$ indefinitely, whereas for $D \approx \sqrt{2}$ (the maximum distance that two nodes could attain), the vacation is immediate, i.e. after one iteration. 


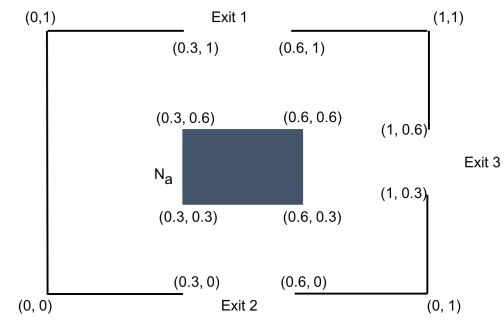

Figure 8: The room described in section 5.2.

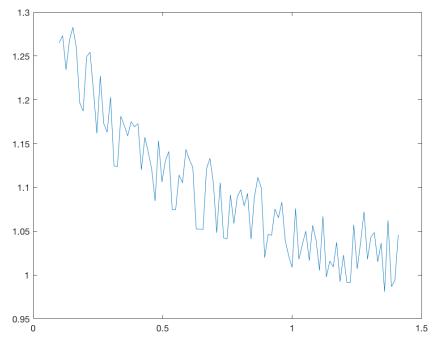

Figure 9: The average vacating iteration time of the room with a no area $N_{a}=[0.3,0.6] \times$ $[0.3,0.6]$ versus different values of the minimum distance $D$.

Subsequently, we considered the same room with a no area $N_{a}=[0.3,0.6] \times$ $[0.3,0.6]$, as depicted in figure 8 , where three new random networks were created to avoid any node inside $N_{a}$. The same experiment was carried out and figure 9 depicts the average exiting time iteration versus the distance $D$. As expected it can be seen that it decreases in a less marked manner due to the existence of $N_{a}$.

We subsequently evaluated the $\mathbf{D}_{\mathbf{k}}\left(G_{k}\right)$ for the above two cases as depicted in figures 10, for a threshold $D=0.2$. Again, the presence of $N_{a}$ results in higher disorder levels.

Finally, figure 11 depicts the average values of $\mathbf{D}_{\mathbf{k}}\left(G_{k}\right)$ for values of $\delta=0.7$, $\delta=0.5$, and $\delta=0.3$ defined by the network in the scenario depicted in figure 7 . As can be seen, the lower the values for $\delta$, the weaker mutual connections are, which results in higher disorder levels and a less efficient solution. 

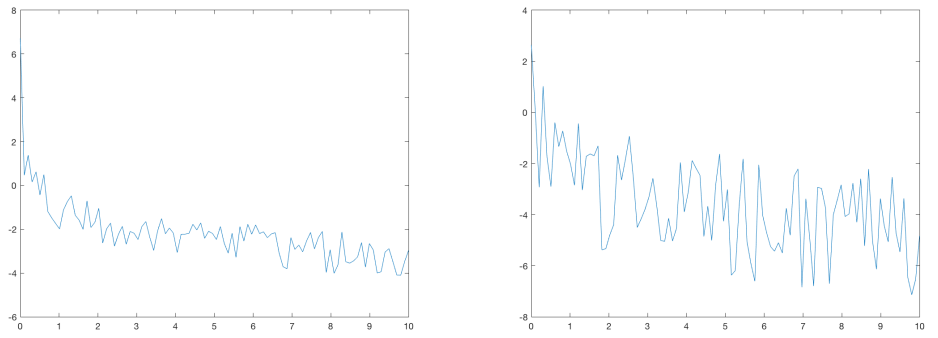

Figure 10: The average values of $\mathbf{D}_{\mathbf{k}}\left(G_{k}\right)$ for the case depicted in figures 7 and 8 , respectively.

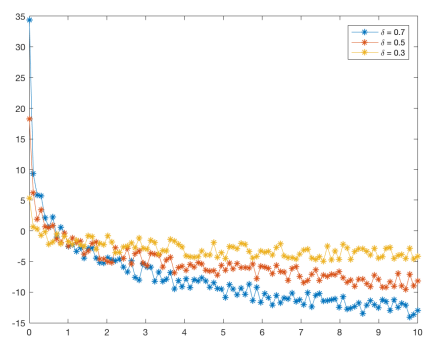

Figure 11: The average values of $\mathbf{D}_{\mathbf{k}}\left(G_{k}\right)$ for the case depicted in figure 7 for values of $\delta=0.7$, $\delta=0.5$, and $\delta=0.3$.

\subsection{Evaluation on the dataset introduced in [14]}

In the last part of the evaluation, we considered the dataset created $^{1}$ in [14] by installing a large network cameras in train stations to capture the full trajectories of pedestrians as depicted in figure 12. The aim of this part of the validation is to capture some general properties of this real-world dataset, rather than providing a full predictive model.

The dataset consists of a number of files, and each of them is a collection of trajectories captured on the same date. Furthermore, each line has the following structure

year-month-dayThour:minute: seconds:milliseconds; Place; $; y$; trackID

where

\footnotetext{
${ }^{1}$ http://www.ivpe.com/crowddata.htm
} 


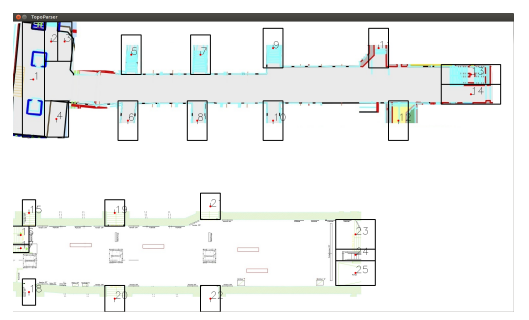

Figure 12: This image illustrates one of the monitored corridors as in [14]. Several cameras are utilised to track, at any given time, the occupancy of the space, where each corridor is approximately $100 \mathrm{~m}$ wide.

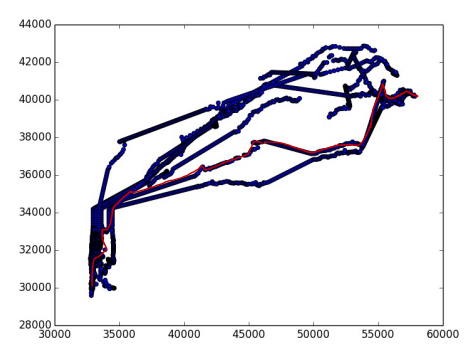

Figure 13: The trajectory of an individual (highlighted in red) with its followers. The initial position is on the right and the final one is on the left hand side.

- year-month-dayThour and minute:seconds:milliseconds refer to the time of the observation

- Place is the location

- $\mathrm{x} ; \mathrm{y}$ are the coordinates in millimetres. All datas have a top-left absolute origin as depicted in figure 12

- trackID uniquely identifies individuals' itineraries.

The dataset was pre-processed to create a set of csv files with the above information. A suitable network was then defined based on whether individuals were close enough. This was assumed to be $2 \mathrm{~m}$.

First of all, we considered a trajectory of a single individual and its followers, as depicted in figure 13. Using equation 18, the time snapshots of the 


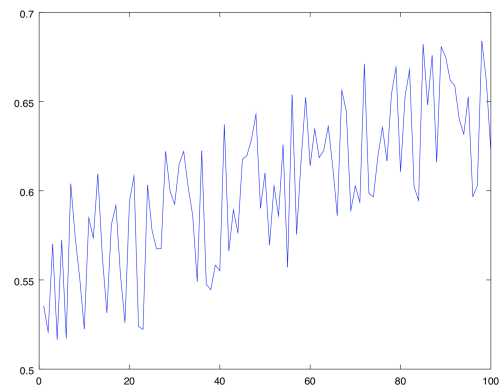

Figure 14: The values of $L_{k}(x)$, evaluated by (18), where $x$ is the individual highlighted in figure 13 , and $k=0, \ldots, 100$
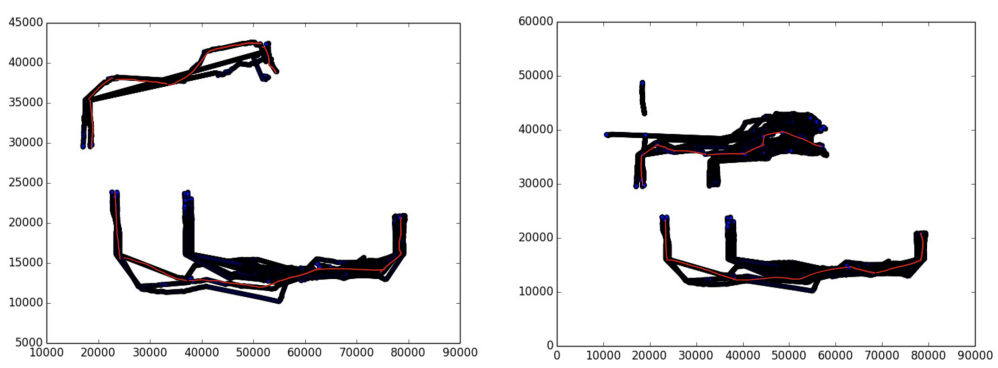

Figure 15: The itineraries of two individuals, where $\delta_{x, y}=0.5$ in the top graph and $\delta_{x, y}=1$, in the bottom one. Note that in this case, the starting points are on the left and the ending points are on the right hand side.
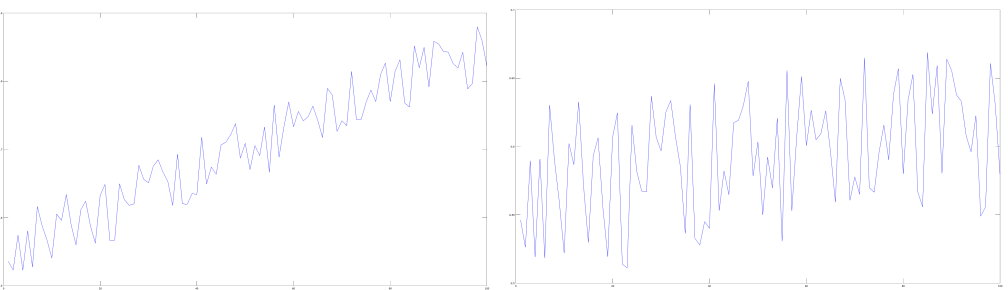

Figure 16: The values of $L_{k}$, evaluated by (18), for the itineraries depicted in figure 15 .

corresponding level of leadership were evaluated, as shown in figure 14, which confirms that such individual indeed exhibits increasing levels of leadership.

Figure 15 depicts a similar scenario. However, in this case we have identified two individuals and their followers have been identified via equation 16, where $\delta_{x, y}=0.5$ in the top graph and $\delta_{x, y}=1$, in the bottom one. 


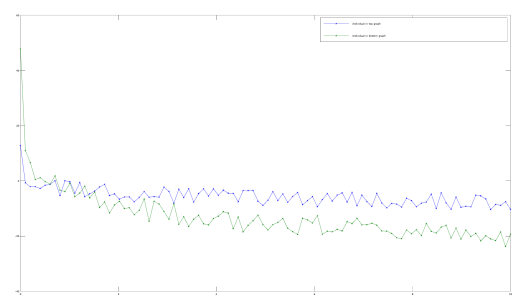

Figure 17: The values of $\mathbf{G}_{\mathbf{k}}\left(G_{t}\right)$, evaluated by (22), for the itineraries depicted in figure 15 .

Figures 16 and 17 show the time snapshots of the values of $L_{k}$ and $\mathbf{G}_{\mathbf{k}}\left(G_{t}\right)$ (as per (22)) respectively, which correspond to the itineraries depicted in figure 15. These are consistent with the fact that the individual at the top clearly exhibits stronger levels of leaderships compared to the bottom one.

\section{Conclusion}

In this article, we have discussed the initial implementation of a system based on the dynamical properties associated with specific topological features of the corresponding topology. The evaluation, which has been carried out, consisted of a combination of mathematical simulation, as well as the dataset described in [14]. Although the former is based on a synthetic dataset, and the latter on a general scenario not specifically related to the applications discussed in this article, it demonstrates the potential of our approach, which can have important applications in disaster management, big data, as well and IoT. In future research, we are aiming to consider more specific real-world datasets from social networks, as well as experimental observations. In fact, this is part of ongoing research aiming to develop a theoretical approach to integrate the topological and algebraic properties of networks with suitable IoT platforms to offer an efficient computational approach to crowd analysis in enclosed spaces. 


\section{References}

[1] A. Kirchner, A. Schadschneider, Simulation of evacuation processes using a bionics-inspired cellular automaton model for pedestrian dynamics, Physica A: Statistical Mechanics and its Applications 312 (1) (2002) 260 276. doi:https://doi.org/10.1016/S0378-4371(02)00857-9.

URL http://www.sciencedirect.com/science/article/pii/ S0378437102008579

[2] F. Piccialli, A. Chianese, Cultural heritage and new technologies: trends and challenges, Personal and Ubiquitous Computing 21 (2) (2017) 187-189. doi:10.1007/s00779-016-0984-y.

URL https://doi.org/10.1007/s00779-016-0984-y

[3] I.-J. Ding, J.-T. Liu, Three-layered hierarchical scheme with a kinect sensor microphone array for audio-based human behavior recognition, Computers and Electrical Engineering 49 (Supplement C) (2016) 173 183. doi:https://doi.org/10.1016/j.compeleceng.2015.03.032.

URL http://www.sciencedirect.com/science/article/pii/ S0045790615001251

[4] N. Bessis, C. Dobre (Eds.), Big Data and Internet of Things: A Roadmap for Smart Environments, Vol. 546, Springer International Publishing, Cham, 2014. doi:10.1007/978-3-319-05029-4.

URL http://dx.doi.org/10.1007/978-3-319-05029-4

[5] B. K. Tannahill, M. Jamshidi, System of systems and big data analytics-bridging the gap, Computers and Electrical Engineering 40 (1) (2014) 2 - 15, 40th-year commemorative issue. doi:https://doi.org/10.1016/j.compeleceng.2013.11.016.

URL http://www.sciencedirect.com/science/article/pii/ S004579061300298X

[6] E. Asimakopoulou, N. Bessis, Buildings and crowds: Forming smart cities 
for more effective disaster management., in: I. You, L. Barolli, F. Tang, F. Xhafa (Eds.), IMIS, IEEE Computer Society, 2011, pp. 229-234.

[7] M. Trovati, J. Hayes, F. Palmieri, N. Bessis, Automated extraction of fragments of bayesian networks from textual sources, Applied Soft Computing 60 (Supplement C) (2017) 508 - 519. doi:https://doi.org/10.1016/j.asoc.2017.07.009.

[8] M. Moussaïd, D. Helbing, G. Theraulaz, How simple rules determine pedestrian behavior and crowd disasters., Proceedings of the National Academy of Sciences of the United States of America, 2011, pp. 6884-6888.

URL http://doi.org/10.1073/pnas.1016507108

[9] J. Was, R. Luba, Towards realistic and effective agent-based models of crowd dynamics, Neurocomputing 146 (Supplement C) (2014) 199 - 209, bridging Machine learning and Evolutionary Computation (BMLEC) Computational Collective Intelligence. doi:https://doi.org/10.1016/j.neucom.2014.04.057.

URL http://www.sciencedirect.com/science/article/pii/ S0925231214007838

[10] A. Turner, A. Penn, Encoding natural movement as an agent-based system: An investigation into human pedestrian behaviour in the built environment, Environment and Planning B: Planning and Design 29 (4) (2002) 473-490. arXiv:https://doi.org/10.1068/b12850, doi:10.1068/b12850.

URL https://doi.org/10.1068/b12850

[11] B. A. Whitehead, James j. gibson: The ecological approach to visual perception. boston: Houghton mifflin, 1979, 332 pp, Behavioral Science 26 (3) (1981) 308-309. doi:10.1002/bs.3830260313.

URL http://dx.doi.org/10.1002/bs.3830260313

[12] K. Nishinari, K. Sugawara, T. Kazama, A. Schadschneider, D. Chowdhury, Modelling of self-driven particles: Foraging ants and pedestrians, Physica A: Statistical Mechanics and its Applications 
372 (1) (2006) 132 - 141, common Trends in Traffic Systems. doi:https://doi.org/10.1016/j.physa.2006.05.016.

URL http://www.sciencedirect.com/science/article/pii/ S0378437106005917

[13] F. Amato, A. Castiglione, A. D. Santo, V. Moscato, A. Picariello, F. Persia, G. Sperla, Recognizing human behaviours in online social networks, Computers and Securitydoi:https://doi.org/10.1016/j.cose.2017.06.002.

URL http://www.sciencedirect.com/science/article/pii/ S0167404817301219

[14] A. Alahi, V. Ramanathan, F.-F. Li, Socially-aware large-scale crowd forecasting., in: CVPR, IEEE Computer Society, 2014, pp. 2211-2218.

[15] E. Pournaras, J. Nikolic, P. Vel=ásquez, M. Trovati, N. Bessis, D. Helbing, Self-regulatory information sharing in participatory social sensing, EPJ Data Science 5 (1) (2016) 14. doi:10.1140/epjds/s13688-016-0074-4.

URL https ://doi.org/10.1140/epjds/s13688-016-0074-4

[16] M. Trovati, Reduced topologically real-world networks: a big-data approach, International Journal of Distributed Systems and Technologies 6 (2) (2015) 132 - 141. doi:https://doi:10.4018/IJDST.2015040102.

[17] M. Trovati, N. Bessis, An influence assessment method based on cooccurrence for topologically reduced big data sets, Soft Computing 20 (5) (2016) 2021-2030. doi:10.1007/s00500-015-1621-9.

URL https://doi.org/10.1007/s00500-015-1621-9

[18] T. Li, H. Chang, M. Wang, B. Ni, R. Hong, S. Yan, Crowded scene analysis: A survey, IEEE Trans. Circuits Syst. Video Techn. 25 (2015) 367-386. 


\section{BIOs}

Dr Marcello Trovati

He is a Reader in Computer Science at Edge Hill University, UK and he has worked for the Universities of Derby and Coventry, and IBM Research. He is involved in several research themes and projects focusing on Mathematical Mod-

elling, Data Science, Big Data Analytics, Network Theory, Machine Learning, Data and Text Mining.

\section{Dr Eleana Asimakopoulou}

She has a PhD (Loughborough University, UK) in managing natural disasters using Grid technology. She is a reviewer in several international conferences and journals and her research interests include emergency management, response and planning for disasters, business continuity, and advanced ICT methods for disaster management.

Prof Nik Bessis

He is a full Professor of Computer Science and the Head of the Department of Computer Science at Edge Hill University, UK. His research is on social graphs for network and big data analytics as well as on developing data push and resource provisioning services in IoT, FI and clouds. 